\title{
Expression of pattern recognition receptors in liver biopsy specimens of children chronically infected with HBV and HCV
}

\author{
Iwona Mozer-Lisewska ${ }^{1}$, Arleta Kowala-Piaskowska ${ }^{1}$, Anna Mania ${ }^{2}$, \\ Renata Jenek ${ }^{3}$, Husam Samara ${ }^{3}$, Elżbieta Kaczmarek ${ }^{4}$, Jan Sikora ${ }^{3}$, \\ Wojciech Służewski ${ }^{2}$, Jan Żeromski ${ }^{3}$
}

\author{
${ }^{1}$ Chair and Department of Infectious Diseases, University of Medical Sciences, Poznan, Poland \\ ${ }^{2}$ Department of Infectious Diseases and Child Neurology, University of Medical Sciences, \\ Poznan, Poland \\ ${ }^{3}$ Chair and Department of Clinical Immunology, University of Medical Sciences, Poznan, Poland \\ ${ }^{4}$ Chair of Pathomorphology, Department of Bioinformatics, University of Medical Sciences, \\ Poznan, Poland
}

\begin{abstract}
Pattern recognition receptors (PRRs) constitute a pivotal arm of innate immunity. Their distribution is widespread and not limited to cells of the immune system. Following our previous findings concerning the expression of Toll-like receptors (TLRs) 2, 3 and 4 in chronic viral hepatitis $C$ of children, we wished to search for other PRRs, including other TLRs, NOD-like receptors (NLRs) and RIG-1-like helicase receptors (RLR) in infected hepatocytes. Liver biopsy fragments from ten children with chronic hepatitis B and C were used and two others in which hepatotropic virus infection was excluded. Frozen sections of liver samples were subjected to ABC immunohistochemistry (IHC) following incubation with a set of antibodies. Results of IHC findings were screened for correlation with clinical/laboratory data of patients. It was found that several PRRs could be shown in affected hepatocytes, but the incidence was higher in hepatitis C than in B. In hepatitis C, TLR1, 2, 4, NALP and RIG-1 helicase showed the most marked expression. In hepatitis B, TLR1, 3, 9, NOD1 and NALP expression were the most conspicuous. Expression PRRs in liver from hepatitis of unknown origin was much lower. It was also the case in cytospins from human hepatoma cell line. Several correlations between PRRs expression and clinical findings in patients could be shown by statistical exploration. In conclusion, this data suggests some role for PRRs in the pathogenesis of chronic viral hepatitis. (Folia Histochemica et Cytobiologica 2011; Vol. 49, No. 3, pp. 410-416)
\end{abstract}

Key words: pattern recognition receptors, viral hepatitis, liver biopsy, children, immunohistochemistry

\section{Introduction}

Hepatotropic viruses, both hepatitis B virus (HBV) and hepatitis $\mathrm{C}$ virus (HCV), remain important pathogenic agents of man, including children. Both viruses

Correspondence address: J. Żeromski, Department of Clinical Immunology, University of Medical Sciences, Rokietnicka Str. 5D, 60-806 Poznan, Poland; tel.: (+ 48 61) 85471 72, fax: (+ 48 61) 8547173 ; e-mail: jzeromski@ump.edu.pl rarely induce acute hepatitis in young patients. Symptomless periods of infection quite often lead, however, to the chronic stage of the disease with several consequences such as liver cirrhosis and hepatocellular carcinoma [1]. The pathogenesis of chronic viral hepatitis is relatively well known. Viruses induce an adaptive immune response in the infected host, which however is usually unable to eradicate them from the body. Activation of the immune effector mechanisms results in liver tissue damage with subsequent proliferation of connective tissue ensuing in 
cirrhosis. Both viruses are apparently not cytopathic $[2,3]$. The inefficiency of adaptive immune response in dealing with viral infection shifted interest toward innate immune mechanisms. The latter dominate in invertebrate animals, being successful against various infections. They were shown to be active in human beings and to influence the course of human diseases, including viral hepatitis [4-6].

Pattern recognition receptors (PRRs) are considered now to play a pivotal role in innate immunity. They are expressed predominantly on cells of the immune system, but have been also shown on various epithelia, including hepatocytes [7, 8]. PRRs are expressed on immune cells, especially on antigen-presenting ones (APC), and participate in the induction of adaptive immunity. Ligands recognized by PRRs are the molecules present on or in pathogens such as viruses, bacteria, fungi and protozoa, being essential for their growth and survival. They include various complex lipids, sugars, nucleic acids, collectively termed pathogen-associated molecular patterns (PAMPs). PRRs comprise several families including Toll-like receptors (TLRs), NOD - nucleotide binding oligomerization domain like receptors (NLRs) and Rig-like helicase receptors (RLR). The best known are TLRs, subdivided now into 11 subgroups in man, according to the specificity of recognized PAMPs. NLRs are relatively newly described intracellular PRRs, consisting of at least 22 members, activated mainly by various bacterial peptidoglycans. RLRs are also intracellular and participate in antiviral defense by promoting type I interferon (IFN) synthesis [9-11].

We have shown three TLRs, namely TLR2, 3 and 4 in liver biopsy tissue sections of children chronically infected with HCV [8]. In the current study, we wanted to learn whether other TLRs, some NLRs and RLRs are also expressed in infected hepatocytes of children suffering from both chronic HBV and HCV hepatitis. Furthermore, we wished to seek a possible relationship between PRRs expression and clinical data of infected children. It will be shown, albeit on a small group of patients, that some links between PRR expression and clinical status of patients really do exist.

\section{Material and methods}

Patients. They were five children, all boys, chronically infected with HBV, aged from six to 14 years, (median age 9.6 years.). Their diagnosis was confirmed both by serological and molecular criteria. Another five children had chronic hepatitis $\mathrm{C}$, including four boys and one girl, aged from eight to 17 years (median age 14.4 years). HCV infection was based on the criteria as above. All ten patients were subjected to percutaneous liver biopsy in order to establish advancement of disease. Informed consent for this procedure was obtained either from parents or the children themselves. Clinical and laboratory data of the patients is shown in Table 1.

Apart from the infected patients, two other boys of comparable age were tested along with those shown above, in which HBV and HCV infection was excluded, but liver biopsy was performed for diagnostic purposes. In the first boy, chronic hepatitis of unknown origin was diagnosed. In the second, a diagnosis of autoimmune hepatitis was finally established. In addition, cell sediments from human hepatoma cell line were tested in parallel. These three specimens were later called 'others'.

$\mathrm{ABC}$ immunohistochemistry. In brief, fragments of liver tissue obtained during biopsy were snap-frozen in dry iceacetone slurry and stored at $-70^{\circ} \mathrm{C}$ until used. Cryostat $4 \mu \mathrm{m}$ sections from liver blocks were then cut $(\mathrm{n}=4-5)$, positioned on each microscopic slide, air-dried, fixed for $5 \mathrm{~min}$ in the cold acetone. Endogenous peroxidase was abolished by the treatment of sections with methanol with $0.6 \%$ hydrogen peroxide for 20 minutes. To avoid non-specific staining, sections were incubated with $1.5 \%$ normal goat serum for one hour.

Primary goat anti-particular PRR antibody was then applied and sections were incubated overnight in a humid chamber in the cold. Primary goat anti-PRRs antibodies used were all from Santa Cruz Biotechnology, CA, USA, and were the same as used previously [12]. The next day, sections were washed thrice with cold PBS and thereafter subjected to biotinylated donkey anti-goat $\operatorname{IgG}$ for $30 \mathrm{~min}$. After washing, the next incubation was with avidin solution, followed by biotinylated horseradish peroxidase (HRP). After subsequent washing steps, substrate solution containing hydrogen peroxide and diaminobenzidine (DAB) was applied. After washing, sections were counterstained with Meyer hematoxylin and embedded in glycerogel. For the whole $\mathrm{ABC}$ procedure, a commercial kit (Santa Cruz) was used. Negative control reactions included:

- omitting primary antibody and using normal goat serum instead;

- performing the reaction on sections of normal human liver (from surgery).

Both control reactions came out completely negative.

As a positive control reaction, frozen sections of human tonsil were run in parallel. This was of limited value, because several PRRs could not be demonstrated in lymphoid cells.

Additionally, cell cytospins of human hepatoma (Hep-62) cell line were subjected to the same immunohistochemical procedure as above. Primary antibodies applied were the same as those used for tissue sections.

Evaluation of immunohistochemical reactions. At least ten microscopic fields at magnification $\times 400$ were analyzed visually and scored under microscope by two independent 
observers. Intensity of positive staining and the number of positive cells were estimated semiquantitatively in numerical scores from 0 to 3: 0 - lack of positive cells; 1 - up to $10 \%$ of positive cells; $2-11-50 \%$ of positive cells; $3-$ $51-100 \%$ of positive cells.

Statistical analysis. Differences between sums of numerical PRRs scores in individual patients of $\mathrm{HBV}, \mathrm{HCV}$ and the 'other group' was tested by ANOVA and post-hoc test of minimal significant difference between results. In order to search for correlation between clinical/laboratory data of patients, results were converted into sets of parameters of descriptive statistics (mean, standard error, standard deviation, number of results). Statistical analysis was performed by means of precise non-parametric tools such as Mann-Whitney test, and Spearman's correlation coefficient. For final statistics, a StatXact (Cytel Co.) program was applied.

\section{Results}

All infected children, both $\mathrm{HCV}^{+}$and $\mathrm{HBV}^{+}$, manifested viremia in the blood. Values of hepatic enzymes (ALT and AST) were raised in almost all patients, but the rise was higher in the $\mathrm{HBV}^{+}$group. Histology of biopsy specimens showed weakly expressed changes in the majority of patients classified as G1S1. All children, with the exception of one (K.P.), were before antiviral therapy. Clinical and laboratory data of all patients tested is set out in Table 1.

When frozen sections of biopsy liver tissue fragments were subjected to PRR antibodies and ABC immunohistochemistry (IHC), the results varied from patient to patient and from one PRR to another (Table 2). In general, the number of PRR positive liver samples and number of scores in individual cases were higher in hepatitis $C$ than in hepatitis $B$ biopsies. Within the TLRs family, approximately the same receptors were most frequently expressed in both diseases tested. They included TLR1, 2, 3, 4, 9 and 10. In the NLR group, all receptors tested, namely NOD1, NOD2, NALP1, Cryopiryn (NALP3) and CARD12, were more strongly expressed in hepatitis $\mathrm{C}$ compared to hepatitis $\mathrm{B}$ liver sections. In the RNL group represented by two receptors, RIG-1 and MDA-5, the former predominated, mainly in hepatitis $\mathrm{C}$ samples. Examples of IHC staining are shown in composite Figure $1(\mathrm{~A}-\mathrm{H})$. As can be seen in Table 2, mean values of numerical scores were higher in hepatitis $\mathrm{C}$ than in hepatitis B for almost all PRRs tested. The mean of the so called 'control group' was lower than those of both examined groups. The only exception was the mean of cryopiryn (NALP3) in which the mean in hepatitis B was lower than in the 'control' group.

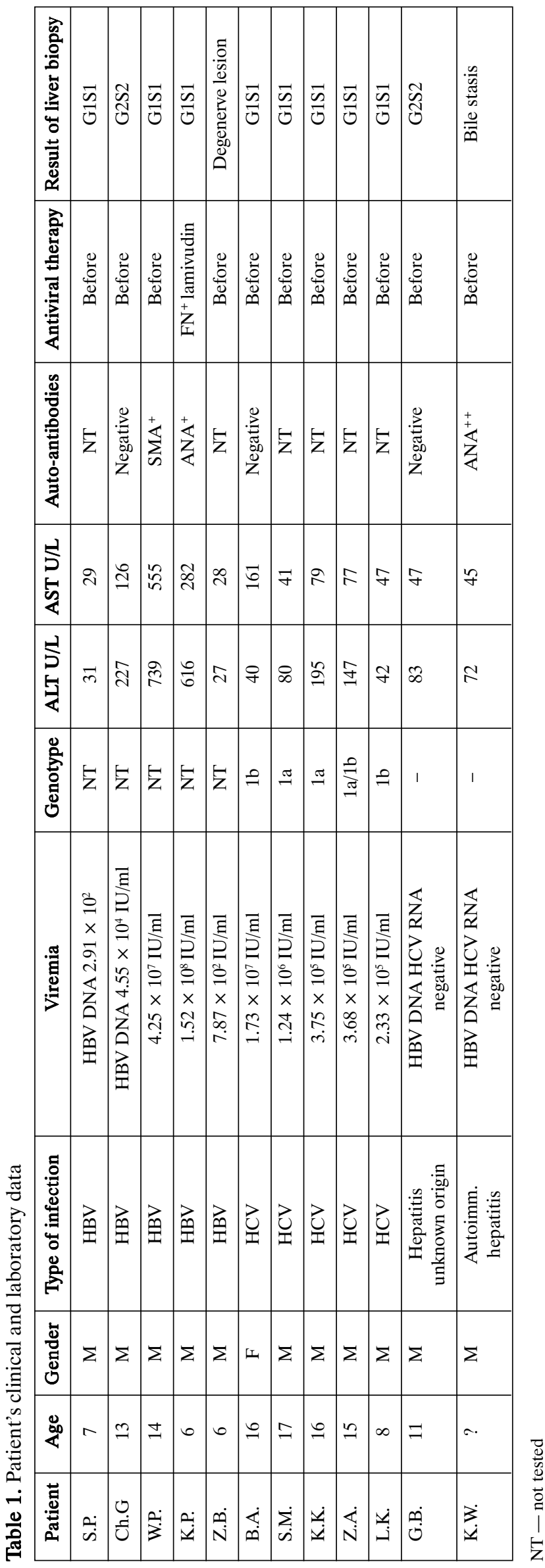




\begin{tabular}{|c|c|c|c|c|c|c|c|c|c|c|c|c|c|c|c|c|c|c|c|}
\hline 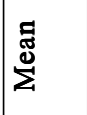 & & $\stackrel{0}{\circ}$ & $\stackrel{0}{-}$ & $\grave{o}$ & $\stackrel{n}{-1}$ & $\stackrel{\infty}{\rightarrow}$ & $\begin{array}{l}\text { ح̂ } \\
\text { ॥ } \\
\text { w }\end{array}$ & & $\stackrel{\circ}{i}$ & $\stackrel{n}{\rightarrow}$ & 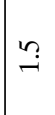 & $\stackrel{t}{-}$ & $\stackrel{\bullet}{-}$ & $\begin{array}{l}0 \\
\infty \\
\text { II } \\
\omega\end{array}$ & & 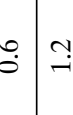 & \begin{tabular}{c|c}
4 \\
-4 & 2
\end{tabular} & & $\begin{array}{l}\underset{i}{i} \\
\| \\
w\end{array}$ \\
\hline$\sum_{\substack{n \\
\Sigma}}^{\infty}$ & & 0 & $\sim$ & 0 & - & 0 & : & & 0 & 0 & 0 & - & 0 & $\tilde{O}$ & & 00 & $\circ-$ & - & ? \\
\hline 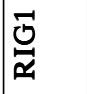 & & 0 & $\alpha$ & 0 & - & 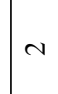 & $\stackrel{\overbrace{}}{\stackrel{-}{2}}$ & & - & $\sim$ & $\mathrm{d}$ & - & $\sim$ & • & & $\begin{array}{lll}-1 & 0\end{array}$ & 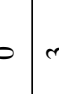 & r & $\stackrel{?}{?}$ \\
\hline 会 & & 0 & - & - & N & - & $\stackrel{\leftrightarrow}{-}$ & & $\alpha$ & $N$ & $\alpha$ & - & 0 & $\stackrel{+}{\sim}$ & & -0 & $0-$ & - & $\tilde{o}$ \\
\hline 完态 & & 0 & - & - & N & - & $\stackrel{\leftrightarrow}{-}$ & & $\alpha$ & $\mathrm{N}$ & $a$ & - & - & ن. & & --1 & -10 & v & ?ִ \\
\hline 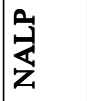 & & 0 & $\mathrm{~N}$ & - & N & N & $\stackrel{\Xi}{-}$ & & $m$ & 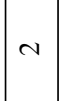 & - & N & $\sim$ & $\stackrel{\leftrightarrow}{i}$ & & $-d$ & $v$ & b & $\stackrel{\circ}{\rightarrow}$ \\
\hline $\begin{array}{l}\text { م̂ } \\
\text { O } \\
\text { z }\end{array}$ & & - & 0 & 0 & - & - & $\stackrel{\circ}{\circ}$ & & $\alpha$ & 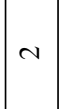 & $\sim$ & - & - & & & 00 & $\circ 0$ & & $\stackrel{8}{0}$ \\
\hline $\begin{array}{l}\overline{0} \\
\text { z }\end{array}$ & & $\bar{\sigma}$ & -1 & - & N & N & $\stackrel{n}{-1}$ & & a & $\sim$ & $a$ & 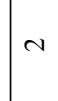 & $\sim$ & $\stackrel{\vec{i}}{\mathrm{i}}$ & & \begin{tabular}{l|l}
0 & $\mathrm{~N}$
\end{tabular} & v & - & $\stackrel{\circ}{\rightarrow}$ \\
\hline 总 & $\oplus$ & 0 & $\mathrm{~N}$ & 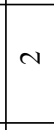 & $m$ & $m$ & $\stackrel{\leftrightarrow}{i}$ & u & $\alpha$ & - & $\mathrm{N}$ & $\bar{z}$ & $m$ & $\stackrel{\leftrightarrow}{i}$ & & $-d$ & v & b & $\stackrel{\circ}{\rightarrow}$ \\
\hline ฉ્ష & 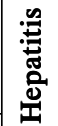 & - & $m$ & - & $\sim$ & $m$ & $\stackrel{\leftrightarrow}{i}$ & 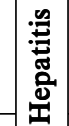 & $m$ & -1 & $\sim$ & 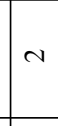 & $\sim$ & $\stackrel{\stackrel{i}{i}}{ }$ & $\begin{array}{l}\text { 泀 } \\
\text { ठ }\end{array}$ & 0 o & v & - & $\stackrel{0}{-}$ \\
\hline 离 & & - & -7 & 占 & - & N & $\stackrel{9}{-1}$ & & $\alpha$ & - & - & - & $\sim$ & $\stackrel{+}{\rightarrow}$ & & \begin{tabular}{l|l}
0 & $\mathrm{~N}$
\end{tabular} & $v$ & $b$ & $\tilde{0}$ \\
\hline 㱐 & & - & $\alpha$ & 0 & $\sim$ & $\alpha$ & $\stackrel{\nexists}{ت}$ & & - & - & - & $N$ & $\sim$ & $\stackrel{-}{\rightarrow}$ & & $0-1$ & -10 & b & $\stackrel{3}{3}$ \\
\hline 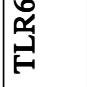 & & 0 & 0 & $\overline{\mathbf{z}}$ & 0 & 0 & 8 & & $\alpha$ & 0 & 0 & - & 0 & : & & $0-1$ & -10 & D & o. \\
\hline 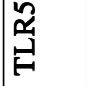 & & 0 & $\sim$ & 0 & 0 & - & $\stackrel{\circ}{\circ}$ & & - & 0 & 0 & - & - & : & 0 & 0 & b & & $\stackrel{8}{\circ}$ \\
\hline 䓵 & & - & - & - & $\sim$ & $m$ & & & $m$ & $\sim$ & $\mathrm{N}$ & $\alpha$ & $\sim$ & ĩ & & $\Rightarrow \quad N$ & $v e$ & b & $\stackrel{\circ}{\longrightarrow}$ \\
\hline 発 & & $N$ & $m$ & - & - & $\alpha$ & $\stackrel{\infty}{\rightarrow}$ & & 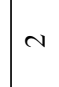 & $\sim$ & $\sim$ & - & $\sim$ & $\stackrel{\infty}{\rightarrow}$ & & $-d$ & $v$ & & $\stackrel{\circ}{\rightarrow}$ \\
\hline$\underset{Z}{2}$ & & - & $\alpha$ & - & $\sim$ & $a$ & $\underset{-}{\stackrel{\sim}{-1}}$ & & $m$ & $m$ & 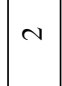 & - & $\mathrm{N}$ & त̃ & & $\begin{array}{lll}N & N\end{array}$ & $\mathrm{v}$ & b & $\stackrel{?}{\rightarrow}$ \\
\hline $\begin{array}{l}\vec{x} \\
\vec{\beta}\end{array}$ & & - & $m$ & - & $\sim$ & $m$ & $\stackrel{\leftrightarrow}{i}$ & & $m$ & $m$ & $\sim$ & 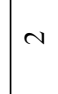 & $m$ & 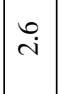 & & $-d$ & $v$ & $b$ & $\stackrel{\circ}{\rightarrow}$ \\
\hline 菢 & & هं & $\begin{array}{l}\dot{\dot{U}} \\
\dot{\Xi}\end{array}$ & 苗 & |ن & $\stackrel{\oplus}{\mathbf{N}}$ & 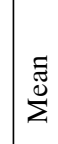 & & 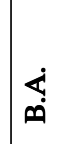 & $\sum_{i=1}^{i}$ & $\dot{u}$ & $\dot{\mathbf{S}}$ & $\ddot{\dot{u}}$ & $\stackrel{\Xi}{\Sigma}^{\Xi}$ & 0 & $\overbrace{}^{\circ}$ & \begin{tabular}{l|l}
3 \\
3 \\
4 \\
4
\end{tabular} & 产 & $\stackrel{\Xi}{\Xi ँ}^{\stackrel{\Xi}{\Sigma}}$ \\
\hline
\end{tabular}



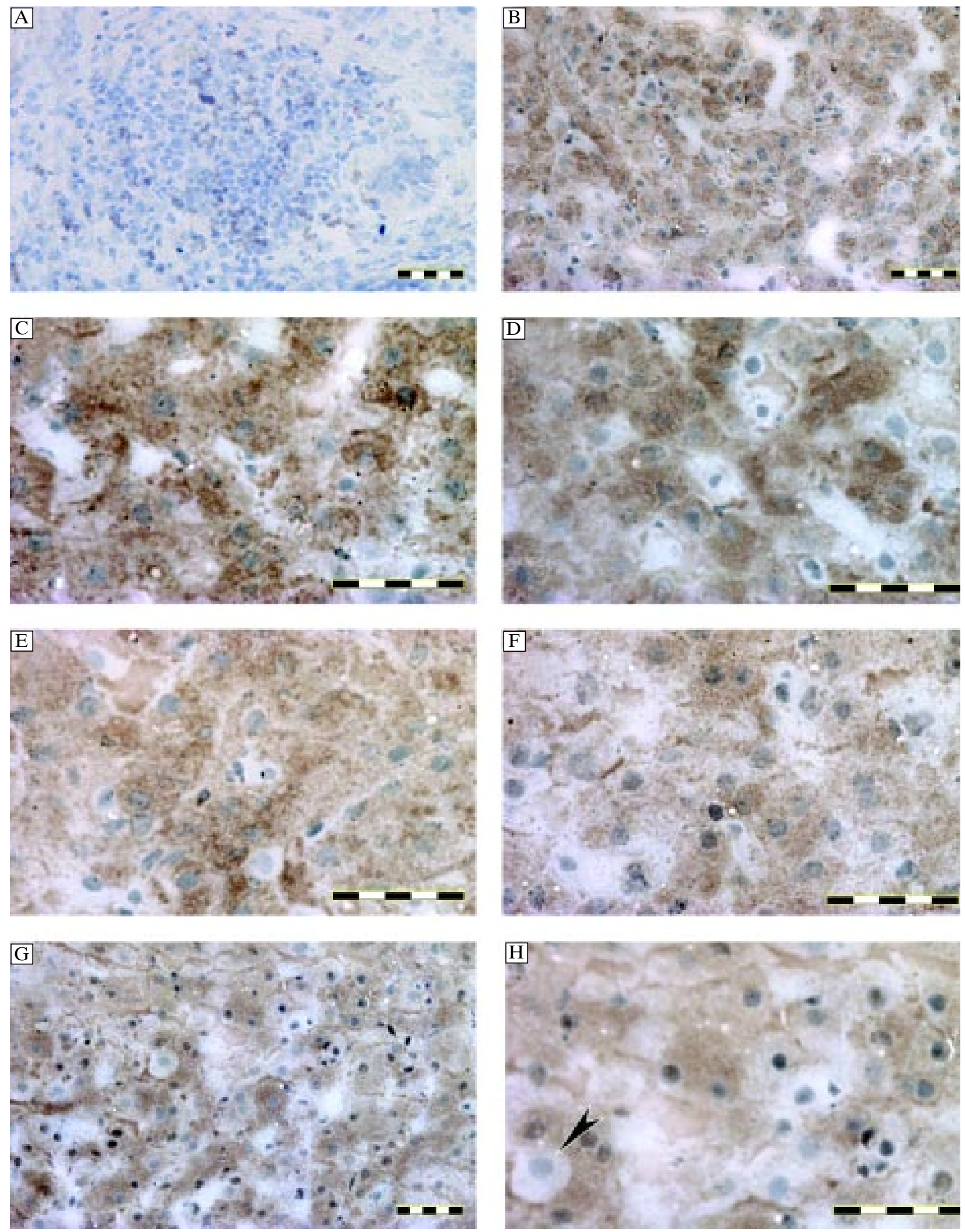

Figure 1. A. Frozen section of liver from $\mathrm{HBV}^{+}$patient. Abundant inflammatory infiltrate is seen. No positive staining in surrounding hepatocytes. ABC reaction but instead of primary antibody- PBS (control); B. As above, but primary Ab anti TLR7. Cytoplasmic reaction in most hepatocytes; C. Frozen section of liver from $\mathrm{HCV}^{+}$patient. Ab anti TLR9. Most liver cells show intensive cytoplasmic staining; D. As above, but Ab anti TLR10. Only some hepatocytes are positive; E. As above, but Ab anti NOD1. Diffuse cytoplasmic reaction in most hepatocytes; F. As above, but Ab anti NALP1. Irregular cytoplasmic staining of hepatocytes; G. As above, but Ab anti CARD12. Majority of cells are positive; H. As above, but higher magnification. Irregular distribution of positive and negative cells; Bar $-50 \mu \mathrm{m}$ 
The numerical scores of all PRRs for individual patients were also added and divided by the number of PRRs tested. The sum of these values in hepatitis $\mathrm{B}(\mathrm{n}=5)$ was compared to that in hepatitis $\mathrm{C}$. It turned out that the sum of hepatitis B was lower than that of hepatitis $C$ (6.2 vs. 8.0). When these values were subjected to ANOVA and post-hoc statistics, the difference between HBV and HCV sums was not significant, but between HCV and the 'others' it was $(\mathrm{p}=0.020)$. There was no visible correlation between total PRR counts and clinical/laboratory data in $\mathrm{HBV}^{+}$patients. In the $\mathrm{HCV}^{+}$group, however, the highest combined PRR value was noticed in the patient (BA) with the highest viremia.

The so-called 'others' group included one hepatitis of unknown origin, $\mathrm{HBV}$ and $\mathrm{HCV}$ negative. Its liver specimens were largely negative or weakly positive with all anti-PRR antibodies. The second liver sample, later diagnosed as autoimmune hepatitis, came out positive or weakly positive with most of the antibodies tested. The third 'others' sample, hepatoma cell line (Hep-62) cytospins, were negative with the exception of Cryopiryn and Rig-1 antibodies.

\section{Evaluation of clinical/laboratory data and $P R R$ values by statistical analysis}

An attempt was made to search for the relationships between clinical/laboratory data and results of PRR by IHC. The following correlations were observed:

1. Significant difference of NOD2 expression between hepatitis B and C ( $p=0.03)$;

2. Borderline significant difference of NOD1 expression between hepatitis B and C $(p=0.08)$;

3. Borderline significant difference of TLR 4 expression between hepatitis B and C $(\mathrm{p}=0.08)$;

Correlation between various parameters tested and viremia was assessed jointly for hepatitis B and hepatitis $\mathrm{C}$ group;

4. Significant correlation was shown between viremia and ALT activity $(\mathrm{r}=0.68, \mathrm{p}=0.02)$;

5. Significant correlation was shown between viremia and AST activity $(r=0.82, p=0.005)$;

6. Significant correlation was demonstrated between viremia and TLR3 expression $(r=-0.64, p=0.04)$;

7. Borderline significance was noted between viremia and CARD12 expression $(\mathrm{r}=0.53, \mathrm{p}=0.07)$;

8. Borderline significance was also noted between viremia and cryopiryn $(r=0.55, p=0.06)$.

\section{Discussion}

The results presented here show that human hepatocytes infected either with HBV or HCV express sev- eral PRRs. In hepatitis B, the most frequent incidence of TLRs included TLR1, 2, 3, 4, 9 and 10, but also NOD1 and NALP1. In hepatitis C, the same PRRs appeared the most frequently, but also NOD2, Cryopiryn (NALP3) and RIG1- like helicase could be demonstrated. It is of interest that hepatocytes from an autoimmune hepatitis case have shown a similar pattern of PRR expression to those of a chronic viral one. This suggests that PRRs ligands may be analogous in both pathological conditions. The reason for such vast PRR expression in these diseases is unknown and any attempts of explanation can only be speculative. Wei et al. [7] have found however, that TLR4 expression in hepatocytes positively correlated with grading scores. In the current study, it was not possible to pinpoint any rules governing PRR expression in individual liver specimens. PRRs do not recognize only PAMPs i.e. molecular patterns of various pathogens. Some of them recognize however products of metabolism or degradation of the body such as uric acid, apoptotic bodies, heat shock proteins and others, collectively termed 'Danger Associated Molecular Patterns' (DAMPs). It is likely that several metabolic end-products formed intracellularly in infected hepatocytes may be recognized by intracellular PRRs such as NLRs. It is known that some NLRs such as NALP3 form intracellular multimolecular complex with caspase-1 termed inflammasome involved in the regulation of secretion of proinflammatory cytokines such as IL-1 [10]. It certainly might trigger an inflammatory reaction against DAMPs. The latter are apparently abundant in liver cells due to the high metabolic turnover in this organ. Activators of NLRs are poorly understood. It is known, for example, that NALPs, members of the NLR family, may be triggered by hypotonic stress, possibly due to the release or activation of some 'danger signal' [13-16]. Such danger signals may originate from cell degradation product following viral infection. NLRs were so far known to be triggered by bacterial peptidoglycans, components of bacterial cell wall. The data from the current study indicates that they can be also triggered by other molecules, not necessarily of bacterial origin.

Higher expression of PRRs in hepatitis C samples compared to HBV ones, although not significant, suggests some hints for this conundrum. As shown in Table 2, means of almost all PRRs were higher in the former group. The sums of numerical values of individual patients divided by the number of PRRs tested were also higher in the HCV group. One possible explanation of the difference in the PRR expression in infected hepatocytes by HCV as compared to HBV ones may be the median age of affected children. The $\mathrm{HBV}$ group was younger than the HCV one. This may 
imply some immaturity of the immune system and some metabolic processes within cells governing PRRs responses both to viral and own wasted products corresponding to PAMPs and DAMPs. Recently new roles for PRR NLRs in the liver have been proposed. It has been shown that hepatocytes express functional NOD1 and NOD2. Stimulation of NOD1 with its ligand (C12-iEDAP) resulted in NF-kappa B activation, CCL5 (RANTES) and CXCL1 chemokine production [17]. In hepatitis $\mathrm{C}$, it was postulated that HCV can subvert PRRs mediated control of adaptive immunity dependent on dendritic cells [18]. Components of RLR family were less frequently demonstrated in examined biopsies. Nevertheless, RIG-1 like helicase was seen in two cases of hepatitis B and in three ones of hepatitis C, while MDA-5 was evident in only one hepatitis B specimen. This may correspond to the relatively low inflammatory intensity in most examined liver tissues as depicted by grading and staging (G1S1) scores. On the other hand, it is known that RLRs and in particular RIG-1 helicase participate in triggering type I interferon secretion following viral infection [19]. It has been shown recently by Hoffman et al. that RIG-1 polymorphism has an influence on its activity during $\mathrm{HCV}$ infection [20].

In spite of the small number of patients, we decided to search for possible correlations between PRR expression and some clinical/laboratory data, being aware of its constraints. Unexpectedly, some correlations could be noticed, either significant or borderline. The former included higher expression of NOD2 in hepatitis $\mathrm{C}$ compared to $\mathrm{B}$ and relatively frequent incidence of TLR3 in both hepatidines. The latter finding may be the reflection of viral etiology of both diseases and TLR 3 propensity for nucleic acids. It was further supported by the presence of significant correlation between TLR3 and viremia. TLR3 has also shown borderline correlation with two NLR members, namely CARD12 (caspase recruitment domain) and cryopiryn (NALP3). The reasons for this are obscure, but it is well known that caspase activation is associated with apoptosis (hepatocytes?).

In conclusion, it appears tempting to postulate that PRRs play some role in the pathogenesis of viral hepatitis $\mathrm{B}$ and $\mathrm{C}$. This is supported even by their vast expression in the hepatocytes of an affected liver, and virtually total absence, at least by IHC screening, from normal ones.

\section{Acknowledgements}

This work was supported by grant nr NN 401174733 from the Polish Ministry of Science and Higher Education (to I M-L).

\section{References}

1. Guido M, Rugge M, Jara P et al. Chronic hepatitis C in children: the pathological and clinical spectrum. Gastroenterology. 1998;115:1525-1531.

2. Lok AS, McMachon BJ. Chronic hepatitis B. Hepatology. 2001;34:1225-1241.

3. Rehermann B, Nascimbeni M. Immunology of hepatitis B and hepatitis C virus infection. Nat Rev Immunol. 2005;5:215-229.

4. Gardy JL, Lynn DJ, Brinkman FSL, Hancock REW. Enabling a systems biology approach to immunology: focus on innate immunity. Feature review. Trends Immunol. 2009; 30:249-262.

5. Takeuchi O, Akira S. Innate immunity to virus infection. Immunol Rev. 2009;227:75-86.

6. Mencin A, Kluwe J, Schwabe RF. Toll-like receptors as targets in chronic liver diseases. Gut. 2009;58:704-720.

7. Wei XQ, Guo YW, Liu JJ et al. The significance of Toll-like 4 (TLR4) expression in patients with chronic hepatitis B. Clin Invest Med. 2008;31:E123-E130.

8. Mozer-Lisewska I, Slużewski W, Kaczmarek M et al. Tissue localization of Toll-like receptors in biopsy specimens of liver from children infected with hepatitis C virus. Scand J Immunol. 2005;62:407-412.

9. Mortinon F, Tschopp J. NLRs join TLRs as innate sensors of pathogens. Trends Immunol. 2005;26:447-454.

10. Creagh EM, O'Neill LAJ. TLRs, NLRs and RLRs: a trinity of pathogen sensors that co-operate in innate immunity. Trends Immunol. 2006;27:352-357.

11. Fukata M, Vamadevan AS, Abreu MT. Toll-like receptors (TLRs) and Nod-like receptors (NLRs) in inflammatory disorders. Semin Immunol. 2009;21:242-253.

12. Sikora J, Frydrychowicz M, Kaczmarek M et al. TLR receptors in laryngeal carcinoma - immunophenotypic, molecular and functional studies. Folia Histochem Cytobiol. 2010;48:624-631.

13. Eisenbarth SC, Flavell RA. Innate instruction of adaptive immunity revisited: the inflammasome. EMBO Mol Med. 2009;2:92-98.

14. Mortinon F, Burns K, Tschopp J. The inflammasome: a molecular platform triggering activation of inflammatory caspases and processing of proIL-beta. Mol Cell. 2002;10:417-426.

15. Mortinon F. Detection of immune danger signals by NALP3. J Leucoc Biol. 2008;83:507-511.

16. Inohara N, Nunez G. NODs: intracellular proteins involved in inflammation and apoptosis. Nat Rev Immunol. 2003; 3:371-382.

17. Scott MJ, Chen C, Sun Q, Billiar TR. Hepatocytes express functional NOD1 and NOD2 receptors: a role for NOD1 in hepatocyte CC and CXC chemokine production. J Hepatol. 2010;53:693-701.

18. Rodrigue-Gervais IG, Lamarre D. Hepatitis C virus subverts pattern recognition receptors - mediated control of adaptative immunity orchestrated by dendritic cells. (In French). Med Sci (Paris). 2010;26: 869-874

19. Foy E, Li K, Sumpter R Jr, Loo YM et al. Control of antiviral defenses through hepatitis $\mathrm{C}$ virus disruption of retinoic acidinducible gene-I signaling. Proc Natl Acad Sci USA. 2005; 102:2986-2991.

20. Hoffman F et al. Proceedings of TOLL - 2008: Recent Advances in Pattern Recognition. Cascais, Portugal, September 24-27, 2008;Abstract 29:p.54.

Submitted: 17 November, 2010 Accepted after reviews: 4 Februrary, 2011 